Bull. Chem. Soc. Ethiop. 2013, 27(3), 421-426.

Printed in Ethiopia

ISSN 1011-3924

DOI: http://dx.doi.org/10.4314/bcse.v27i3.11

(c) 2013 Chemical Society of Ethiopia

\title{
A CONVENIENT THREE-COMPONENT SYNTHESIS OF CARBAMATOALKYL NAPHTHOLS CATALYZED BY CERIUM AMMONIUM NITRATE
}

\author{
Min Wang ${ }^{1 *}$, Yang Liu ${ }^{1}$, Zhiguo Song ${ }^{2}$ and Shuang Zhao ${ }^{1}$ \\ ${ }^{1}$ College of Chemistry, Chemical Engineering and Food Safety, Bohai University, Jinzhou \\ 121013, China \\ ${ }^{2}$ Management Center for Experiment, Bohai University, Jinzhou 121013, China
}

(Received October 2, 2012; revised February 25, 2013)

\begin{abstract}
A highly efficient synthesis of carbamatoalkyl naphthols has been performed by a one-pot threecomponent condensation of 2-naphthol, aldehydes, and methyl/ethyl/benzyl carbamates in the presence of cerium ammonium nitrate under solvent-free conditions at $70{ }^{\circ} \mathrm{C}$. The solvent, optimal amounts of raw materials and catalyst, and reaction temperature are investigated. Experimental results show that only $0.1 \mathrm{mmol}$ catalyst is enough to induce the conversion. Most reactions are performed within a short reaction time. The structures of all products were characterized by IR, ${ }^{1} \mathrm{H} \mathrm{NMR},{ }^{13} \mathrm{C} \mathrm{NMR}$, MS, and elemental analysis. A mechanism to rationalize the reaction has been proposed.
\end{abstract}

KEY WORDS: Carbamatoalkyl naphthols, Cerium ammonium nitrate, One-pot synthesis, Solvent-free conditions

\section{INTRODUCTION}

Compounds bearing 1,3-amido oxygenated functional groups are ubiquitous to a variety of biologically important natural products and potent drugs including a number of nucleoside antibiotics and HIV protease inhibitors such as ritonavir, lipinavir, and the hypotensive [1]. In addition, 1-carbamatoalkyl-2-naphthols can convert to 1-aminomethyl-2-naphthols by a carbamate hydrolysis reaction. 1-Aminomethyl-2-naphthols have been reported to show cardiovascular activity [2].

Though carbamatoalkyl naphthols are very important, only a few literatures focused on their synthesis have been published. The reported catalysts include triethylbenzylammonium chloride [3], $\mathrm{SiO}_{2}-\mathrm{NaHSO}_{4}$ [4], Brønsted-acidic ionic liquids [5], silica-supported polyphosphoric acid [6], silica-supported Preyssler nano particles [7], $\mathrm{SiO}_{2}-\mathrm{HClO}_{4}$ [8], Zwitterionic-type molten salt [9], $\left[\mathrm{MeC}(\mathrm{OH})_{2}\right]^{+} \mathrm{ClO}_{4}^{-}[10]$, ionic liquid $\left[\mathrm{NMP}{ }^{+} \mathrm{HSO}_{4}^{-}[11]\right.$, sulfamic acid functionalized magnetic nanoparticles [12], $\mathrm{Mg}\left(\mathrm{OOCCF}_{3}\right)_{2}$ [13], [Dsim] $\mathrm{HSO}_{4}$ [14], and $\left[\mathrm{Et}_{3} \mathrm{~N}_{-} \mathrm{SO} \mathrm{O}_{3} \mathrm{H}\right] \mathrm{Cl}$ [15], etc. However, most of these methods suffer from drawbacks including low yields, expensive reagents and catalysts. Therefore, the development of high yielding and less expensive catalytic method is desired.

Cerium ammonium nitrate (CAN) has been widely used in organic transformations due to its many advantages such as high reactivity, commercial availability, ease of handling, and stability in different solvents. We herein describe the catalytic activity of CAN for the efficient threecomponent synthesis of carbamatoalkyl naphthols (4a-r) under solvent-free conditions (Scheme $1)$.

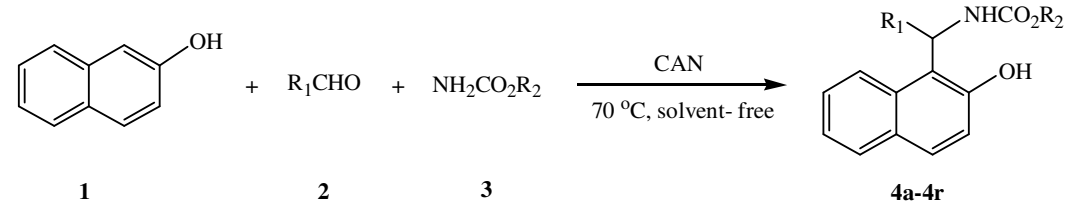

Scheme 1. Three-component synthesis of carbamatoalkyl naphthols catalyzed by CAN.

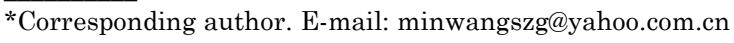


General

\section{EXPERIMENTAL}

Melting points were determined using a RD-II micromelting point apparatus. Infrared spectra were recorded on a Scimitar 2000 series Fourier transform instrument of Varian. ${ }^{1} \mathrm{H}$ NMR and ${ }^{13} \mathrm{C}$ NMR spectra were recorded on an Agilent 400-MR instrument in DMSO- $d_{6}$ using TMS as an internal standard. Mass spectra were obtained with an Agilent 1100 series LC/MSD VL ESI instrument. All the reagents were analytical grade and were purchased from the Shanghai Chemical Reagent Company. TLC was performed on silica gel polygram SIL G/UV 254 plates.

Typical procedure for the synthesis of carbamatoalkyl naphthols (4)

To a mixture of $\beta$-naphthol ( $5 \mathrm{mmol})$, an aldehyde $(5 \mathrm{mmol})$, and a carbamate $(5.5 \mathrm{mmol})$, CAN $(0.1 \mathrm{mmol})$ was added. The reaction mixture was magnetically stirred on a preheated water bath at $70{ }^{\circ} \mathrm{C}$. After completion of the reaction (monitored by TLC), the reaction mixture was cooled to r.t., washed with $\mathrm{H}_{2} \mathrm{O} / \mathrm{EtOH}(\mathrm{v} / \mathrm{v}=1 / 1)$, and the residue was recrystallized from $\mathrm{H}_{2} \mathrm{O} / \mathrm{EtOH}$ $(\mathrm{v} / \mathrm{v}=2 / 3)$. The products were characterized by m.p., IR, ${ }^{1} \mathrm{H}$ NMR, ${ }^{13} \mathrm{C}$ NMR and elemental analysis. Spectral data for new compounds are given below.

\section{Methyl ((2-chlorophenyl)(2-hydroxynaphthalen-1-yl)methyl) carbamate (4e)}

White solid. IR (KBr): 3431, 3220, 1690, 1527, 1341, 1051, 819, 753, $706 \mathrm{~cm}^{-1}$; ${ }^{1} \mathrm{H}$ NMR (400 MHz, DMSO- $\left.d_{6}\right): \delta=9.96(\mathrm{~s}, 1 \mathrm{H}, \mathrm{OH}), 8.04(\mathrm{~d}, 1 \mathrm{H}, J=8.2 \mathrm{~Hz}, \mathrm{NH}), 7.86(\mathrm{~d}, 1 \mathrm{H}, J=7.1 \mathrm{~Hz}$, ArH), $7.77(\mathrm{dd}, 2 \mathrm{H}, J=17.4,8.6 \mathrm{~Hz}, \mathrm{ArH}), 7.52(\mathrm{~d}, 1 \mathrm{H}, J=4.6 \mathrm{~Hz}, \mathrm{ArH}), 7.40(\mathrm{dd}, 2 \mathrm{H}, J=$ 12.3, $6.8 \mathrm{~Hz}, \mathrm{ArH}), 7.26(\mathrm{~d}, 3 \mathrm{H}, J=13.9 \mathrm{~Hz}, \mathrm{ArH}), 7.16(\mathrm{~d}, 1 \mathrm{H}, J=8.6 \mathrm{~Hz}, \mathrm{ArH}), 6.91(\mathrm{~d}, 1 \mathrm{H}$, $J=7.8 \mathrm{~Hz}, \mathrm{CH}), 3.54\left(\mathrm{~s}, 3 \mathrm{H}, \mathrm{OCH}_{3}\right) ;{ }^{13} \mathrm{C}$ NMR $\left(100 \mathrm{MHz}\right.$, DMSO- $\left.d_{6}\right): \delta=156.5,153.9,139.8$, 133.0, 132.9, 130.3, 129.9, 129.7, 129.0, 128.8, 128.7, 126.9, 126.7, 123.3, 122.7, 119.0, 117.4, 51.9, 50.1; MS (EI, $70 \mathrm{eV}): \mathrm{m} / z(\%) 340$ [(M-H), 100$]$. Anal. calcd. for $\mathrm{C}_{19} \mathrm{H}_{16} \mathrm{NO}_{3} \mathrm{Cl}: \mathrm{C}, 66.77$; H, 4.72; N, 4.10. Found: C, 66.89; H, 4.64; N, 4.17.

Methyl ((2,4-dichlorophenyl)(2-hydroxynaphthalen-1-yl)methyl) carbamate (4g)

White solid. IR (KBr): 3403, 3260, 1678, 1519, 1062, 874, 753, $718 \mathrm{~cm}^{-1}$; ${ }^{1} \mathrm{H}$ NMR (400 MHz, DMSO-d6): $\delta=9.97(\mathrm{~s}, 1 \mathrm{H}, \mathrm{OH}), 8.02(\mathrm{~d}, 1 \mathrm{H}, \mathrm{J}=8.5 \mathrm{~Hz}, \mathrm{NH}), 7.96(\mathrm{~d}, 1 \mathrm{H}, \mathrm{J}=7.8 \mathrm{~Hz}, \mathrm{ArH})$, $7.78(\mathrm{dd}, 2 \mathrm{H}, \mathrm{J}=17.8,8.7 \mathrm{~Hz}, \operatorname{ArH}), 7.57-7.38(\mathrm{~m}, 4 \mathrm{H}, \mathrm{ArH}), 7.28(\mathrm{t}, 1 \mathrm{H}, \mathrm{J}=7.3 \mathrm{~Hz}, \mathrm{ArH})$, $7.14(\mathrm{~d}, 1 \mathrm{H}, \mathrm{J}=8.7 \mathrm{~Hz}, \mathrm{ArH}), 6.85(\mathrm{~d}, 1 \mathrm{H}, \mathrm{J}=8.0 \mathrm{~Hz}, \mathrm{CH}), 3.55\left(\mathrm{~s}, 3 \mathrm{H}, \mathrm{OCH}_{3}\right) ;{ }^{13} \mathrm{C} \mathrm{NMR}(100$ MHz, DMSO- $\left.d_{6}\right): \delta=156.6,153.9,139.2,133.5,133.0,132.4,131.6,130.1,129.0,128.9$, 128.6, 127.0, 126.9, 123.1, 122.8, 118.9, 116.7, 52.0, 49.8; MS (EI, $70 \mathrm{eV}): \mathrm{m} / \mathrm{z}$ (\%) 375 [(M$\left.\mathrm{H})^{-}, 100\right]$. Anal. calcd. for $\mathrm{C}_{19} \mathrm{H}_{15} \mathrm{NO}_{3} \mathrm{Cl}_{2}$ : C, 60.66; H, 4.02; N, 3.72. Found: C, 60.51; H, 4.07; $\mathrm{N}, 3.61$.

Ethyl ((2-nitrophenyl)(2-hydroxynaphthalen-1-yl)methyl) carbamate (4l)

White solid. IR (KBr): 3402, 3277, 1686, 1531, 1334, 1039, 813, 744, $697 \mathrm{~cm}^{-1} ;{ }^{1} \mathrm{H}$ NMR (400 MHz, DMSO- $\left.d_{6}\right): \delta 9.79(\mathrm{~s}, 1 \mathrm{H}, \mathrm{OH}), 7.92(\mathrm{~d}, 1 \mathrm{H}, J=8.6 \mathrm{~Hz}, \mathrm{NH}), 7.81-7.72(\mathrm{~m}, 4 \mathrm{H}, \mathrm{ArH})$, 7.61-7.58 (m, 2H, ArH), $7.44(\mathrm{dt}, 2 \mathrm{H}, J=7.5,6.5 \mathrm{~Hz}, \mathrm{ArH}), 7.29-7.26(\mathrm{~m}, 2 \mathrm{H}, \mathrm{ArH}), 7.05(\mathrm{~d}$, $1 \mathrm{H}, J=8.7 \mathrm{~Hz}, \mathrm{CH}), 4.04\left(\mathrm{q}, 2 \mathrm{H}, J=7.0 \mathrm{~Hz}, \mathrm{CH}_{2}\right), 1.15\left(\mathrm{t}, 3 \mathrm{H}, J=7.0 \mathrm{~Hz}, \mathrm{CH}_{3}\right) ;{ }^{13} \mathrm{C} \mathrm{NMR}$ $\left(100 \mathrm{MHz}, \mathrm{DMSO}-d_{6}\right): \delta 156.4,154.0,149.0,136.9,133.3,132.5,130.3,129.4,128.8,128.5$, 128.1, 127.0, 124.4, 123.0, 122.8, 118.8, 116.5, 60.5, 48.1, 15.0; MS (EI, $70 \mathrm{eV}): \mathrm{m} / \mathrm{z}$ (\%) 365 [(M-H), 100$]$. Anal. calcd. for $\mathrm{C}_{20} \mathrm{H}_{18} \mathrm{~N}_{2} \mathrm{O}_{5}: \mathrm{C}, 65.57 ; \mathrm{H}, 4.95 ; \mathrm{N}, 7.65$. Found: $\mathrm{C}, 65.71 ; \mathrm{H}$, $4.87 ; \mathrm{N}, 7.72$. 
Ethyl ((4-nitrophenyl)(2-hydroxynaphthalen-1-yl)methyl) carbamate (4m)

White solid. IR (KBr): 3430, 3185, 1685, 1518, 1351, 1049, 821, 739, $708 \mathrm{~cm}^{-1} ;{ }^{1} \mathrm{H}$ NMR (400 MHz, DMSO- $\left.d_{6}\right): \delta=10.21(\mathrm{~s}, 1 \mathrm{H}, \mathrm{OH}), 8.15(\mathrm{~d}, 2 \mathrm{H}, J=8.8 \mathrm{~Hz}, \mathrm{ArH}), 7.94-7.73(\mathrm{~m}, 4 \mathrm{H}, \mathrm{NH}$ and $\mathrm{ArH}), 7.49$ (d, 2H, $J=8.6 \mathrm{~Hz}, \mathrm{ArH}), 7.43(\mathrm{t}, 1 \mathrm{H}, J=7.5 \mathrm{~Hz}, \mathrm{ArH}), 7.30(\mathrm{t}, 1 \mathrm{H}, J=7.5 \mathrm{~Hz}$, $\mathrm{ArH}), 7.23(\mathrm{~d}, 1 \mathrm{H}, J=8.8 \mathrm{~Hz}, \mathrm{ArH}), 6.97(\mathrm{~d}, 1 \mathrm{H}, J=7.7 \mathrm{~Hz}, \mathrm{CH}), 4.07$ (q, 2H, $J=6.7 \mathrm{~Hz}$, $\left.\mathrm{CH}_{2}\right), 1.17\left(\mathrm{t}, 3 \mathrm{H}, J=6.7 \mathrm{~Hz}, \mathrm{CH}_{3}\right) ;{ }^{13} \mathrm{C}$ NMR (100 MHz, DMSO-d $\left.d_{6}\right): \delta=156.7,153.5,151.1$, 146.4, 132.3, 130.3, 129.1, 128.8, 127.5, 127.2, 123.7, 123.2, 123.0, 118.8, 118.4, 60.7, 50.5, 15.0; MS (EI, $70 \mathrm{eV}): \mathrm{m} / z$ (\%) 365 [(M-H), 100$]$. Anal. calcd. for $\mathrm{C}_{20} \mathrm{H}_{18} \mathrm{~N}_{2} \mathrm{O}_{5}: \mathrm{C}, 65.57 ; \mathrm{H}$, 4.95; N, 7.65. Found: C, 65.43; H, 5.02; N, 7.72.

\section{Ethyl ((2,4-dichlorophenyl)(2-hydroxynaphthalen-1-yl)methyl) carbamate (4n)}

White solid. IR (KBr): 3412, 3071, 1683, 1514, 1336, 1052, 815, 743, $721 \mathrm{~cm}^{-1} ;{ }^{1} \mathrm{H}$ NMR (400 MHz, DMSO- $\left.d_{6}\right): \delta=9.93(\mathrm{~s}, 1 \mathrm{H}, \mathrm{OH}), 8.04(\mathrm{~d}, 1 \mathrm{H}, J=8.6 \mathrm{~Hz}, \mathrm{NH}), 7.81-7.75(\mathrm{~m}, 3 \mathrm{H}, \mathrm{ArH})$, $7.58(\mathrm{~d}, 1 \mathrm{H}, J=8.5 \mathrm{~Hz}, \mathrm{ArH}), 7.49(\mathrm{~d}, 1 \mathrm{H}, J=1.5 \mathrm{~Hz}, \operatorname{ArH}), 7.44(\mathrm{t}, 1 \mathrm{H}, J=7.5 \mathrm{~Hz}, \operatorname{ArH}), 7.38$ (dd, $1 \mathrm{H}, J=6.8,1.8 \mathrm{~Hz}, \mathrm{ArH}), 7.28(\mathrm{t}, 1 \mathrm{H}, J=7.4 \mathrm{~Hz}, \mathrm{ArH}), 7.14(\mathrm{~d}, 1 \mathrm{H}, J=8.8 \mathrm{~Hz}, \mathrm{ArH})$, $6.86(\mathrm{~d}, 1 \mathrm{H}, J=8.1 \mathrm{~Hz}, \mathrm{CH}), 3.98\left(\mathrm{q}, 2 \mathrm{H}, J=6.7 \mathrm{~Hz}, \mathrm{CH}_{2}\right), 1.14\left(\mathrm{t}, 3 \mathrm{H}, J=6.4 \mathrm{~Hz}, \mathrm{CH}_{3}\right) ;{ }^{13} \mathrm{C}$ NMR (100 MHz, DMSO- $\left.d_{6}\right): \delta=156.1,154.0,139.3,133.5,132.9,132.3,131.6,130.1,129.0$, 128.9, 128.6, 127.0, 126.9, 123.1, 122.8, 119.0, 116.8, 60.4, 49.7, 15.0; MS (EI, $70 \mathrm{eV}): \mathrm{m} / \mathrm{z}$ (\%) 389 [(M-H) $\left.{ }^{-}, 100\right]$. Anal. calcd. for $\mathrm{C}_{20} \mathrm{H}_{17} \mathrm{NO}_{3} \mathrm{Cl}_{2}$ : C, 61.55; H, 4.39; N, 3.59. Found: C, $61.41 ; \mathrm{H}, 4.44 ; \mathrm{N}, 3.63$.

\section{Benzyl ((2-chlorophenyl)(2-hydroxynaphthalen-1-yl)methyl) carbamate (4q)}

White solid. IR (KBr): 3421, 3170, 1700, 1518, 1335, 1050, 819, 754, $694 \mathrm{~cm}^{-1}$; ${ }^{1} \mathrm{H}$ NMR (400 MHz, DMSO- $\left.d_{6}\right): \delta=9.96(\mathrm{~s}, 1 \mathrm{H}, \mathrm{OH}), 8.04(\mathrm{~d}, 2 \mathrm{H}, J=7.8 \mathrm{~Hz}, \mathrm{ArH}), 7.81-7.75(\mathrm{~m}, 2 \mathrm{H}, \mathrm{NH}$ and $\mathrm{ArH}), 7.52-7.25(\mathrm{~m}, 10 \mathrm{H}, \mathrm{ArH}), 7.16(\mathrm{~d}, 2 \mathrm{H}, J=7.3 \mathrm{~Hz}, \mathrm{ArH}), 6.94(\mathrm{~d}, 1 \mathrm{H}, J=5.8 \mathrm{~Hz}$, $\mathrm{CH}), 5.09\left(\mathrm{~d}, 1 \mathrm{H}, J=12.0 \mathrm{~Hz}, \mathrm{CH}_{2}\right), 5.01\left(\mathrm{~d}, 1 \mathrm{H}, J=12.0 \mathrm{~Hz}, \mathrm{CH}_{2}\right) ;{ }^{13} \mathrm{C} \mathrm{NMR}(100 \mathrm{MHz}$, DMSO- $\left.d_{6}\right): \delta=156.0,153.9,139.7,137.6,133.0,132.9,130.3,129.9,129.7,129.0,128.9$, 128.7, 128.1, 127.8, 126.9, 126.7, 123.3, 122.7, 118.9, 117.3, 65.8, 50.1; MS (EI, $70 \mathrm{eV}): \mathrm{m} / \mathrm{z}$ (\%) $416\left[(\mathrm{M}-\mathrm{H})^{-}, 100\right]$. Anal. calcd. for $\mathrm{C}_{25} \mathrm{H}_{20} \mathrm{NO}_{3} \mathrm{Cl}$ : C, 71.86; H, 4.82; N, 3.35. Found: C, $71.98 ; \mathrm{H}, 4.89 ; \mathrm{N}, 3.27$.

Benzyl ((4-chlorophenyl)(2-hydroxynaphthalen-1-yl)methyl) carbamate (4r)

White solid. IR (KBr): 3402, 3200, 1681, 1515, 1321, 1042, 812, 746, $696 \mathrm{~cm}^{-1}$; ${ }^{1} \mathrm{H}$ NMR (400 MHz, DMSO- $\left.d_{6}\right): \delta=10.15(\mathrm{~s}, 1 \mathrm{H}, \mathrm{OH}), 7.93(\mathrm{~d}, 1 \mathrm{H}, J=8.2 \mathrm{~Hz}, \mathrm{NH}), 7.82-7.77(\mathrm{~m}, 3 \mathrm{H}, \mathrm{ArH})$, 7.39-7.23 (m, 12H, ArH), $6.90(\mathrm{~d}, 1 \mathrm{H}, J=8.1 \mathrm{~Hz}, \mathrm{CH}), 5.11\left(\mathrm{~d}, 1 \mathrm{H}, J=12.6 \mathrm{~Hz}, \mathrm{CH}_{2}\right), 5.01(\mathrm{~d}$, $\left.1 \mathrm{H}, J=12.6 \mathrm{~Hz}, \mathrm{CH}_{2}\right) .{ }^{13} \mathrm{C}$ NMR $\left(100 \mathrm{MHz}, \mathrm{DMSO}-d_{6}\right): \delta=156.5,153.4,141.8,137.3,132.3$, 131.3, 129.9, 129.0, 128.8, 128.7, 128.4, 128.3, 128.2, 127.4, 127.0, 123.7, 123.4, 123.0, 118.9, 118.7, 66.1, 50.3; MS (EI, $70 \mathrm{eV}): \mathrm{m} / z(\%) 416$ [(M-H)', 100]. Anal. calcd. for $\mathrm{C}_{25} \mathrm{H}_{20} \mathrm{NO}_{3} \mathrm{Cl}$ : C, 71.86; H, 4.82; N, 3.35. Found: C, 71.73; H, 4.93; N, 3.39.

\section{RESULTS AND DISCUSSION}

First, in order to optimize the reaction conditions, the effects of solvent, molar ratio of raw materials, amount of catalyst and reaction temperature on the products yields were evaluated by carrying out the model reaction of $\beta$-naphthol 1, 2-nitrobenzaldehyde 2 and methyl carbamate 3 
(Table 1). It was found that the best results were obtained with 0.1 mmol CAN under solventfree conditions at $70{ }^{\circ} \mathrm{C}$ (Table 1, entry 4). The reaction was completed within $1.0 \mathrm{~h}$ and the expected product was obtained in a $91 \%$ yield, while increase the amount of catalyst or evaluate the reaction temperature further would decrease the product yield. A blank experiment of $\beta$ naphthol, 2-nitrobenzaldehyde, and methyl carbamate in the absence of catalyst was investigated. No product was obtained after $4.0 \mathrm{~h}$ (Table 1, entry 13).

Table 1. Condensation of $\beta$-naphthol, 2-nitrobenzaldehyde and methyl carbamate catalyzed by CAN under various conditions ${ }^{\mathrm{a}}$.

\begin{tabular}{|c|c|c|c|c|c|c|}
\hline Entry & Solvent $(\mathrm{mL})$ & $\mathrm{n}_{(\mathbf{1})}: \mathrm{n}_{(2)}: \mathrm{n}_{(3)}$ & CAN $(\mathrm{mmol})$ & Temp. $\left({ }^{\circ} \mathrm{C}\right)$ & Time $(\mathrm{h})$ & Yield $(\%)^{\mathrm{b}}$ \\
\hline 1 & $\mathrm{H}_{2} \mathrm{O}(3)$ & $1: 1: 1.1$ & 0.1 & 70 & 2.0 & 0 \\
\hline 2 & $\mathrm{EtOH}^{\mathrm{b}}(3)$ & $1: 1: 1.1$ & 0.1 & 70 & 1.0 & 6 \\
\hline 3 & $\mathrm{CH}_{3} \mathrm{CN}(3)$ & $1: 1: 1.1$ & 0.1 & 70 & 3.0 & 19 \\
\hline 4 & - & $1: 1: 1.1$ & 0.1 & 70 & 1.0 & 91 \\
\hline 5 & - & $1: 1: 1$ & 0.1 & 70 & 1.0 & 85 \\
\hline 6 & - & $1: 1: 1.2$ & 0.1 & 70 & 1.0 & 92 \\
\hline 7 & - & $1: 1: 1.1$ & 0.025 & 70 & 1.5 & 80 \\
\hline 8 & - & $1: 1: 1.1$ & 0.05 & 70 & 1.3 & 86 \\
\hline 9 & - & $1: 1: 1.1$ & 0.15 & 70 & 1.0 & 88 \\
\hline 10 & - & $1: 1: 1.1$ & 0.1 & 50 & 1.2 & 81 \\
\hline 11 & - & $1: 1: 1.1$ & 0.1 & 60 & 1.0 & 85 \\
\hline 12 & - & $1: 1: 1.1$ & 0.1 & 80 & 1.0 & 82 \\
\hline 13 & - & $1: 1: 1.1$ & 0 & 70 & 4.0 & 0 \\
\hline
\end{tabular}

${ }^{\text {a }} \beta$-naphthol was 5 mmol. ${ }^{\text {b}}$ Yields refer to isolated pure products.

Table 2. Synthesis of carbamatoalkyl naphthols in the presence of CAN without solvent ${ }^{\mathrm{a}}$.

\begin{tabular}{|c|c|c|c|c|c|c|}
\hline Entry & $\mathrm{R}_{1}$ & $\mathrm{R}_{2}$ & Time (h) & Product & Yield (\%) & M.p. $\left({ }^{\circ} \mathrm{C}\right)$ \\
\hline 1 & $\mathrm{Ph}$ & $\mathrm{CH}_{3}$ & 1.5 & $\mathbf{4 a}$ & 85 & $224-225[5]$ \\
\hline 2 & $2-\mathrm{NO}_{2} \mathrm{C}_{6} \mathrm{H}_{4}$ & $\mathrm{CH}_{3}$ & 1.0 & $\mathbf{4 b}$ & 91 & $241-243[5]$ \\
\hline 3 & $3-\mathrm{NO}_{2} \mathrm{C}_{6} \mathrm{H}_{4}$ & $\mathrm{CH}_{3}$ & 0.6 & $\mathbf{4 c}$ & 92 & $244-246[5]$ \\
\hline 4 & $4-\mathrm{NO}_{2} \mathrm{C}_{6} \mathrm{H}_{4}$ & $\mathrm{CH}_{3}$ & 0.7 & $\mathbf{4 d}$ & 92 & $210-211[13]$ \\
\hline 5 & $2-\mathrm{ClC}_{6} \mathrm{H}_{4}$ & $\mathrm{CH}_{3}$ & 1.0 & $\mathbf{4 e}$ & 89 & $218-220$ \\
\hline 6 & $4-\mathrm{ClC}_{6} \mathrm{H}_{4}$ & $\mathrm{CH}_{3}$ & 1.0 & $\mathbf{4 f}$ & 93 & $202-204[5]$ \\
\hline 7 & $2,4-\mathrm{Cl}_{2} \mathrm{C}_{6} \mathrm{H}_{3}$ & $\mathrm{CH}_{3}$ & 1.0 & $\mathbf{4 g}$ & 88 & $210-211$ \\
\hline 8 & $4-\mathrm{CH}_{3} \mathrm{C}_{6} \mathrm{H}_{4}$ & $\mathrm{CH}_{3}$ & 10.0 & $\mathbf{4 h}$ & trace & - \\
\hline 9 & $4-\mathrm{CH}_{3} \mathrm{OC}_{6} \mathrm{H}_{4}$ & $\mathrm{CH}_{3}$ & 10.0 & $\mathbf{4 i}$ & trace & - \\
\hline 10 & $\mathrm{CH}_{3} \mathrm{CH}_{2}$ & $\mathrm{CH}_{3}$ & 10.0 & $\mathbf{4 j}$ & 0 & - \\
\hline 11 & $\mathrm{Ph}_{12}$ & $\mathrm{CH}_{2} \mathrm{CH}_{3}$ & 1.5 & $\mathbf{4 k}$ & 80 & $203-204[11]$ \\
\hline 12 & $2-\mathrm{NO}_{2} \mathrm{C}_{6} \mathrm{H}_{4}$ & $\mathrm{CH}_{2} \mathrm{CH}_{3}$ & 1.5 & $\mathbf{4 l}$ & 85 & $215-216$ \\
\hline 13 & $4-\mathrm{NO}_{2} \mathrm{C}_{6} \mathrm{H}_{4}$ & $\mathrm{CH}_{2} \mathrm{CH}_{3}$ & 0.5 & $\mathbf{4 m}$ & 91 & $229-230$ \\
\hline 14 & $2,4-\mathrm{Cl}_{2} \mathrm{C}_{6} \mathrm{H}_{3}$ & $\mathrm{CH}_{2} \mathrm{CH}_{3}$ & 1.0 & $\mathbf{4 n}$ & 89 & $196-198$ \\
\hline 15 & $\mathrm{Ph}_{16}$ & $\mathrm{CH}_{2} \mathrm{Ph}$ & 2.5 & $\mathbf{4 o}$ & 86 & $185-187[5]$ \\
\hline 16 & $3-\mathrm{NO}_{2} \mathrm{C}_{6} \mathrm{H}_{4}$ & $\mathrm{CH}_{2} \mathrm{Ph}$ & 1.0 & $\mathbf{4 p}$ & 90 & $206-208[5]$ \\
\hline 17 & $2-\mathrm{ClC}_{6} \mathrm{H}_{4}$ & $\mathrm{CH}_{2} \mathrm{Ph}$ & 1.5 & $\mathbf{4 q}$ & 91 & $211-213$ \\
\hline 18 & $4-\mathrm{ClC}_{6} \mathrm{H}_{4}$ & $\mathrm{CH}_{2} \mathrm{Ph}$ & 2.0 & $\mathbf{4 r}$ & 89 & $179-181$ \\
\hline
\end{tabular}

${ }^{\mathrm{a}} \beta$-naphthol $5 \mathrm{mmol}$, aldehydes $5 \mathrm{mmol}$, carbamates $5.5 \mathrm{mmol}$, CAN $0.1 \mathrm{mmol}, 70{ }^{\circ} \mathrm{C}$. ${ }^{\mathrm{b}}$ Yields refer to isolated pure products.

Then, the scope and limitation of the reactions of $\beta$-naphthol $\mathbf{1}$, aldehydes $\mathbf{2}$, and methyl/ethyl/benzyl carbamate $\mathbf{3}$ catalyzed by CAN were studied, the results were shown in 
Table 2. Most reactions proceeded smoothly with good to excellent yields under the optimized conditions. Aryl aldehydes with electron-withdrawing groups proved to be more active than those with electron-donating groups. The position of the substituent on the aromatic ring shows no effect on the product yield. The reaction of aliphatic aldehyde such as propionaldehyde isolated no desired product (Table 2, entry 10). Beside methyl carbamate, ethyl carbamate and benzyl carbamate were also utilized for the condensation, and they all participated well.

Reusability of the catalyst was also investigated. For this purpose, the same model reaction was again studied under optimized conditions. After the completion of the reaction, the reaction mixture was cooled to room temperature, and ethanol aqueous was added. Due to the fact that the catalyst was soluble in ethanol and water, it could therefore be recovered by evaporating the filtrate. The separated catalyst was washed with $\mathrm{CH}_{2} \mathrm{Cl}_{2}$, dried at $50{ }^{\circ} \mathrm{C}$ for $1 \mathrm{~h}$ and reused in another reaction. As shown in Table 3, the catalyst could be reused at least two times without significant loss of activity. However, the reaction time had to prolong due to the loss of the catalyst.

Table 3. The comparison of efficiency of CAN as catalyst in the synthesis of $\mathbf{4 b}$ after three times ${ }^{\mathrm{a}}$.

\begin{tabular}{|c|c|c|c|}
\hline Run & Time $(\mathrm{h})$ & ${\text { Yield }(\%)^{\mathrm{b}}}$ & Amount of catalyst $(\mathrm{mg})$ \\
\hline First & 1.0 & 91 & 54.8 \\
\hline Second & 1.3 & 88 & 39.4 \\
\hline Third & 1.5 & 82 & 21.7 \\
\hline
\end{tabular}

a $\beta$-naphthol $5 \mathrm{mmol}$, 2-nitrobenzaldehyde $5 \mathrm{mmol}$, methyl carbamate $5.5 \mathrm{mmol}$, CAN $0.1 \mathrm{mmol}(54.8 \mathrm{mg}), 70^{\circ} \mathrm{C}$. ${ }^{\mathrm{b}}$ Yields refer to isolated pure products.

We propose a reaction mechanism of the CAN-catalyzed condensation. CAN act as a Lewis acid catalyst that facilitates the formation of $o-\mathrm{MQ}$, which further react with a nucleophile (carbamate) to form the desired carbamatoalkyl naphthols 4 [16].

\section{CONCLUSIONS}

From the results above it can be concluded that CAN is an efficient catalyst for the one-pot three-component condensation of $\beta$-naphthol, aromatic aldehydes (with electron-withdrawing groups) and methyl/ethyl/benzyl carbamates. The new protocol offers several significant advantages such as inexpensive and commercially available catalyst, mild reaction condition, simple work-up and excellent yields (80-93\%). This study enlarges the existing methods for the synthesis of the title compounds.

\section{ACKNOWLEDGEMENT}

This research work was financially supported by the Education Committee of Liaoning Province of China (No. L2011198).

\section{REFERENCES}

1. Dingermann, T.; Steinhilber, D.; Folkers, G. Molecular Biology in Medicinal Chemistry, Wiley-VCH: Weinheim; 2004; p 132.

2. Shen, A.Y.; Tsai, C.T.; Chen, C.L. Eur. J. Med. Chem. 1999, 34, 877.

3. Mosslemin, M.H.; Nateghi, M.R.; Mohebat, R. Monatsh. Chem. 2008, 139, 1247.

4. Shaterian, H.R.; Hosseinian, A.; Ghashang, M. Tetrahedron Lett. 2008, 49, 5804.

5. Tavakoli-Hoseini, N.; Heravi, M.M.; Bamoharran, F.F.; Davoodnia, A. Bull. Korean Chem. Soc. 2011, 32, 787. 
6. Shaterian, H.R.; Hosseinian, A.; Ghashang, M. Chin. J. Chem. 2009, $27,821$.

7. Heravi, M.M.; Tavakoli-Hoseini, N.; Bamoharram, F.F. Green Chem. Lett. Rev. 2010, 3, 263.

8. Shaterian, H.R.; Hosseinian, A.; Ghashang, M. Synth. Commun. 2009, 39, 2560.

9. Kundu, D.; Majee, A.; Hajra, A. Catal. Commun. 2010, 11, 1157.

10. Tamaddon, F.; Bistgani, J.M. Synlett 2011, 20, 2947.

11. Deshmukh, K.M.; Qureshi, Z.S.; Patil, Y.P.; Bhanage, B.M. Synth. Commun. 2012, 42, 93.

12. Yarahmadi, H.; Shaterian, H.R. J. Chem. Res. 2012, 36, 52.

13. Shafiee, M.R.M.; Moloudi, R.; Ghashang, M. J. Chem. Res. 2011, 35, 622.

14. Zare, A.; Yousofi, T.; Moosavi-Zare, A.R. RSC Adv. 2012, 2, 7988.

15. Zare, A.; Akbarzadeh, S.; Foroozani, E.; Kaveh, H.; Moosavi-Zare, A.R.; Hasaninejad, A.; Mokhlesi, M.; Beyzavi, M.H.; Zolfigol, M.A. J. Sulfur Chem. 2012, 33, 259.

16. Song, Z.G.; Zhao, S.; Wan, X. Chin. J. Org. Chem. 2011, 31, 870. 